Graphical abstract

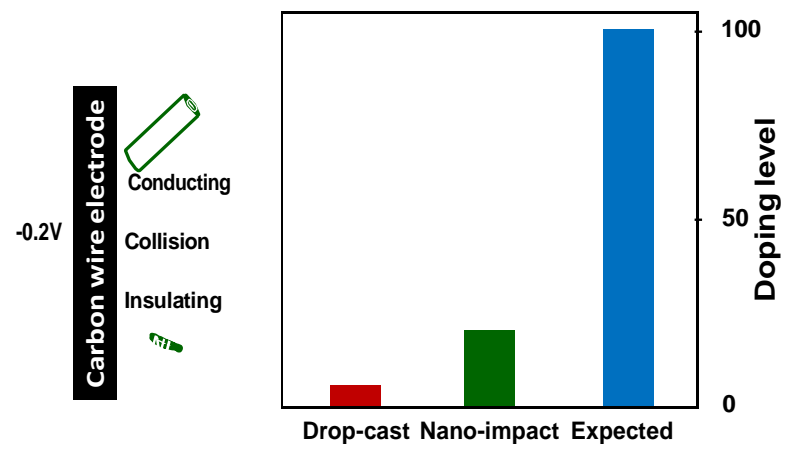




\title{
Quantifying Charge Transfer to Nanostructures: Polyaniline Nanotubes
}

\author{
Jianyun Liu ${ }^{a, b}$, Guodong Zhu ${ }^{a}$, Xiuting Li ${ }^{b}$, Christopher Batchelor-McAuley ${ }^{b}$,
} Stanislav V. Sokolov ${ }^{b}$, Richard G. Compton ${ }^{b *}$

a State Environmental Protection Engineering Center for Pollution Treatment and Control in the Textile Industry, College of Environmental Science and Engineering, Donghua University, Shanghai 201620, P. R. China

b Department of Chemistry, Physical \& Theoretical Chemistry Laboratory, University of Oxford, Oxford OX1 3QZ, United Kingdom

\section{Corresponding Author: *Email: Richard.compton@chem.ox.ac.uk}

\begin{abstract}
The nano-impact method is used to quantify electrolytic doping levels of single polyaniline nanotubes in aqueous sulphuric acid solution. Even at extreme potentials only partial $(\leq 18 \%)$ doping of the tubes is observed. Comparisons are made with the doping of ensembles of tubes where the levels of charge transfer appears even less. The implications for practical applications are assessed.
\end{abstract}

KEYWORDS Polyaniline, nanotube, nano-impact, electrochemical doping, quantification of charge transfer

\section{Introduction}


Polyaniline(PANI) finds widespread applications in a diversity of fields ranging across sensors[1-4], rechargeable batteries[5, 6], supercapacitors[7-10], optoelectric devices[11], corrosion protection[12, 13], anti-electrostatic coatings[14] and magnetic shielding[15], reflecting in part the high levels of conductivity attainable and the chemical stabilities of the material. PANI is formed by the chemical or electrochemical oxidation of aniline. The properties of this material are strongly dependent upon its oxidation and protonation state. The fully oxidised form is known as pernigraniline(Per) and the addition of a total of one electron and one proton per aniline monomer unit leads to the formation of the fully reduced form leucoemeraldine[16]. Hence, for a polymer comprising of $m$ aniline monomers there is a total of $m$ possible oxidation states. However, as evidenced by the UV-Vis spectrum of PANI in N-Methyl-2-pyrrolidone (NMP), at least to a first approximation, one only needs to consider a total of three "allowed" oxidation states[17].The behaviour of the material is described by a repeat unit containing four aniline monomer units (see Fig. 1). This reflects the predominantly localised bonding associated with the material. The dominant oxidation states are pernigraniline (Per, $y=2)$, emeraldine (Em, y = 1) and leucoemeraldine (Leuco, y $=0$ ). Of these structures the technologically most important form is that of emeraldine[18]. The effective $\mathrm{pK}_{\mathrm{a}}$ of emeraldine is $~ 2.5[19]$. Moreover on protonation to form $\mathrm{Em} \cdot \mathrm{HCl}$ the electronic structure of the material is altered, via internal redox chemistry of the polymer, leading to the formation of a semiquinone like structure and the material takes a radical chain structure as evidenced by the associated EPR spectrum[20]. The Em·HCl material is commonly found to have a deep green colour. However the optical properties of the material are sensitive to its crystallinity[17]. Specifically, when $\mathrm{Em} \cdot \mathrm{HCl}$ is deposited from an NMP solution it is a deep purple colour (these crystalline structures are sometimes denoted separately as $\mathrm{Em} \cdot \mathrm{HCl} \mathrm{I}$ and $\mathrm{Em} \cdot \mathrm{HCl} \mathrm{II})$. It is however, this protonated form of the emeraldine $(\mathrm{Em} \cdot \mathrm{HCl} \mathrm{I}$ and II) that exhibits 'metallic' conductivity, where the polymer is approximately 
$10^{10}$ times more conductive than its deprotonated analog(Em base) [19, 21]. It should be commented that the material does not exhibit semi-conductor properties when partially protonated, evidencing that the material forms isolated regions of high and low conductivity. The various oxidation and protonation states of PANI can be readily diagrammatically summarised using a scheme of squares (see Fig. 1) [22, 23]. Here a horizontal movement corresponds to the addition of two-electrons and a vertical shift signifies a change in the protonation state by two-protons. Electrochemically below $\mathrm{pH} 4$ the voltammetry of PANI exhibits two redox waves, the first redox wave corresponds to the oxidation of leucoemeraldine to the protonated form of emeraldine $(\mathrm{Em} \cdot \mathrm{HCl})$. The second voltammetric wave, which is $\mathrm{pH}$ sensitive $\left(\sim 120 \mathrm{mV} \mathrm{pH}^{-1}\right)$, corresponds to the oxidation of $\mathrm{Em} \cdot \mathrm{HCl}$ to the fully oxidised form pernigraniline[16].

Morphologically, PANI nanomaterials have been produced in various forms including nanofibers[24-26], nanospheres[27, 28], and nanotubes[29-31]. The latter provide a high surface area and claimed increased 'accessibility'. Moreover, since this fabrication process can be controlled during synthesis typically via an electrospinning process, the aspect ratio of the tubes as well as their inner and outer diameter can be optimized for various applications[3].

Implicit in the use of optimized structures is that the properties and behaviour of the single nanostructured entity such as an optimised nanotube(or sphere, or fibre) is reflected in the use of ensembles of the structures as utilised in many applications. This is typically difficult to measure requiring measurements on different length (and often time) scales. Thus for example whilst individual nanotubes might show high conductivity, the requirement in ensembles to transfer charge between tubes [32] may lead to significantly altered behaviour which limits or even eliminates the benefits of nanoscale optimisation at the individual entity 
level. In particular for the case of nanoparticles very significant differences between single entities and ensembles have been noted where the aggregated or agglomerated nanoparticles show quantitatively very different behaviours from single nanoparticles[33-35]. One effective and clearcut method for assessing particular charge transfer properties at the single entity level is the nano-impacts method [36-39] in which the entities (nanoparticles or nanotubes) are suspended in a solution containing a microelectrode. From time to time the random nearBrownian motion of the entity brings it into electrical contact with the electrode so that, for the period of impact (collision) electrochemical currents can flow as a result of electron transfer to or from the entity and/or capacitance effects [37, 40].

In the following, we use the nano-impact approach to study the electrochemical doping of single PANI nanotubes and then compare the results with analogous data obtained from the nanotube ensembles formed by drop-casting a large number of tubes onto the surface of a macroelectrode.

\section{Materials and experimental:}

\subsection{Materials}

All reagents were purchased from Sigma-Aldrich at reagent grade and used without further purification. All electrochemical measurements were carried out in a supporting electrolyte of $0.2 \mathrm{M} \mathrm{H}_{2} \mathrm{SO}_{4}$. The electrolytes were degassed with pure nitrogen for $20 \mathrm{~min}$. All aqueous solutions were prepared with deionised water of resistivity not less than 18.2 M $\Omega$ at 298 K (Millipore, Billerica, MA).

\subsection{Fabrication of PANI hollow fibres}

Polyaniline (PANI) hollow fibres were fabricated using a previously described method[3, 28]. Briefly, the self-made electrospun polystyrene (PS) nanofiber with the average size of 
$156 \pm 60 \mathrm{~nm}$ was treated in concentrated sulfuric acid $(98 \%, \mathrm{w} / \mathrm{w})$ followed by rinsing with water to get the sulfonated PS. After impregnation of the sulfonated PS in $150 \mathrm{mM}$ aniline hydrochloride solution overnight, the oxidation polymerisation of aniline results in the formation of PANI in the presence of ammonium persulfate APS[n(APS):n(aniline)=1:1] in 1 M HCl solution. Subsequently, the resulting product was washed with water and ethanol and dried overnight in a vacuum oven at $60{ }^{\circ} \mathrm{C}$. Finally, the inner core of PANI-covered fibre was dissolved by tetrahydrofuran[28] to obtain hollow PANI fibres. After washing with $1 \mathrm{M} \mathrm{HCl}$, the obtained PANI mainly exists in its metallic conductive emeraldine salt form, as evidenced via ${ }^{13} \mathrm{C}$ NMR by MacDiarmid[21]. The detailed characterization of the PANI hollow fibres was performed previously[3].

\subsection{Preparation of the PANI nanotube suspension}

A PANI suspension solution was prepared by mixing $6.4 \mathrm{mg}$ of the hollow fibre with 20 mL pure water and sonicating via a Fisher Scientific ultrasonic bath S60 (230 V 50/60 Hz $150 \mathrm{~W})$ for $60 \mathrm{~min}\left(<40^{\circ} \mathrm{C}\right)$ to break up the long fibres into shorter nanotubes. For nanoimpact experiments, the PANI suspension solution was prepared by mixing $2.0 \mathrm{mg}$ of hollow fibres with $20 \mathrm{~mL}$ of $0.2 \mathrm{M} \mathrm{H}_{2} \mathrm{SO}_{4}$ solution. After sonication for $60 \mathrm{~min}$, it was diluted into $0.01 \mathrm{~g} \mathrm{~L}^{-1}$ with $0.2 \mathrm{M} \mathrm{H}_{2} \mathrm{SO}_{4}$ solution. Before each experiment, the suspension was sonicated 2 min to prevent the aggregation of the nanotube suspension.

\subsection{Carbon fibre micro wire electrode fabrication}

Carbon fibre micro wire electrodes were fabricated with the previous method provided by Ellison et al[41]. First, a metal wire was connected to a carbon fiber (diameter $7.0 \mu \mathrm{m}$, Goodfellow, Cambridge, UK) using silver epoxy (RS Components, Corby, UK) conductive adhesive. Then the adhesive was set after holding it in an oven at approximately $60{ }^{\circ} \mathrm{C}$ for 15 min. Subsequently the wire was threaded through a plastic pipette tip so that only the carbon 
fibre extended out of the end. Cyanoacrylate adhesive was used to seal the interstice between the carbon fibre/metal wire and the plastic pipette tip. After holding the tip in air overnight to set the adhesive, the carbon fibre micro wire electrode was obtained by cutting the exposed carbon fibre to a length of approximately $1 \mathrm{~mm}$.

\subsection{PANI nanotube coated glassy carbon electrode}

A $3.0 \mathrm{~mm}$ diameter glassy carbon electrode (GCE) supplied by $\mathrm{CH}$ instruments (Austin, TX, USA) was used as a working electrode. Before modification, the electrode was polished successively with 1.0, 0.3 and $0.05 \mu \mathrm{m} \mathrm{Al}_{2} \mathrm{O}_{3}$ slurries and was then sonicated in water and ethanol successively for 2 min in each step. The cleaned electrode was dried with nitrogen immediately before use. Different volumes $(1,2,5 \mu \mathrm{L})$ of freshly prepared PANI nanotube suspensions of $0.32 \mathrm{~g} \mathrm{~L}^{-1}$ were drop cast onto a clean GCE surface to get a modified GCE with various coverages. For the higher drop volume of $8 \mu \mathrm{L}$ and $15 \mu \mathrm{L}$, the electrode was drop cast twice using 4 and $7.5 \mu \mathrm{L}$ each time, respectively. After each coating, the electrode was dried under a nitrogen flow.

\subsection{Electrochemical procedures}

Cyclic voltammetry (CV) measurements were recorded using a $\mu$-Autolab III potentiostat (Autolab, Utrecht, Netherlands) with a conventional three-electrode configuration including a PANI drop-cast GCE working electrode, a saturated calomel reference electrode (SCE, BASi, Japan) and a spiral Pt wire counter electrode. The CV experiment was carried out in a nitrogen saturated $0.2 \mathrm{M} \mathrm{H}_{2} \mathrm{SO}_{4}$ aqueous solution at $23 \pm 2{ }^{\circ} \mathrm{C}$. Before each $\mathrm{CV}$ scan, the open circuit potential (OCP) was read and the CV scan started from the OCP to ensure no reduction or oxidation of material prior to each measurement. The average OCP for the modified electrode was found to be $0.415 \pm 0.010 \mathrm{~V}$. The charge quantity was calculated based on the reduction current integration only from the first CV scan of each modified electrode. 
More than three repeats were done for each coverage. Nano-impact chronoamperometry experiments were conducted via the $\mu$-Autolab potentiostat and 3-electrode system as above, but using a carbon fibre micro wire as the working electrode. $0.2 \mathrm{M} \mathrm{H}_{2} \mathrm{SO}_{4}$ containing $0.01 \mathrm{~g}$ $\mathrm{L}^{-1}$ PANI was used as electrolyte in a $10 \mathrm{~mL}$ sample vial. Following bubbling with nitrogen for $20 \mathrm{~min}$, chronoamperometric experiments were run at different potentials. The duration for each scan was $50 \mathrm{~s}$. In the interval between the scans, the suspension was bubbled with nitrogen for $5 \mathrm{~s}$ to ensure a uniform dispersion of PANI nanotubes and to avoid oxygen interfering with the electrochemical reactions. The vial of suspension was sonicated every 20 scans to prevent aggregation of the nanotubes.

\subsection{Morphology characterization}

Transmission electron microscopy (TEM, 2100F, JEOL, Japan) was applied for the characterization of the inner structure of the PANI nanotubes. Scanning electron microscopy (SEM, Hitachi S-4700, Japan, at an acceleration voltage of 5-10 kV) was used to view the morphology of nanotube and to measure the average size of the PANI suspension. An ITO glass slide was used as the substrate. The substrate was cleaned by sonicating in $50^{\circ} \mathrm{C}$ aqueous detergent for $30 \mathrm{~min}$, deionised water for $5 \mathrm{~min}$, and finally acetone $30 \mathrm{~min}$. Finally $5 \mu \mathrm{L}$ of a $0.1 \mathrm{~g} \mathrm{~L}^{-1}$ PANI suspension was drop-cast onto the clean ITO glass slide. After drying in the air, an ultra-thin Au film was sputter-coated on the PANI layer to prevent charging of the specimen.

\section{Results and discussion}

In this section, the morphological characteristics of the PANI nanotube used in this work are first addressed. Then, the redox properties of PANI nanotube ensembles coated on a GCE are studied using cyclic voltammetry. Subsequently, nano-impact chronoamperometry is 
employed to investigate the electrochemical doping of suspended PANI nanotubes in solution with charge transfer from/to the carbon fibre wire electrode. The charge transferred to a single nanotube suspended in solution as measured via chronoamperometry is compared with the ensembles' response on a macroelectrode as measured using cyclic voltammetry. Finally, the influence of the nanotube's electrical conductivity as a function of the different redox states of the PANI is addressed.

\subsection{SEM characterization of PANI nanotubes}

Polyaniline (PANI) nanotubes were fabricated using a previously described method[3, 28]. The full characterization for the PANI hollow fibres has been performed in previous work[3]. Fig. 2A shows the morphology of PANI hollow fibres. PANI presents a continuous fibre structure with a rough texture on the PANI surface. The fibres extend over tens of micrometers in length. The upper left inlay in Fig. 2A is the cross section of the PANI nanotubes. The tubular structure of the PANI nanotubes can be clearly seen with the inner diameter of $\sim 150 \mathrm{~nm}$. It is consistent with the diameter of the PS template (See SI). The TEM image in the lower left inlay shows the hollow structure of PANI tube, proving that the PS template has been removed completely. Fig. 2B exhibits the SEM image of the PANI nanotubes deposited on an ITO substrate. Well dispersed individual PANI nanotubes are observed on the ITO surface. The size analysis, based on 154 samples that were identified from the electron micrographs (Fig. 2B), gives the length (Fig. 2C) and width (Fig. 2D) distribution of the PANI nanotubes. The average length and width were estimated to be 2.89 $\pm 1.9 \mu \mathrm{m}$ and $0.39 \pm 0.06 \mu \mathrm{m}$, respectively. Thus, the average volume of one nanotube $V_{\text {tube }}$ is estimated to be $(2.9 \pm 1.9) \times 10^{-19} \mathrm{~m}^{3}$, assuming the inner diameter of the nanotube is the same as the diameter of the PS template $(156 \pm 30 \mathrm{~nm}$, see SI). This SEM result indicates that the PANI nanotubes can be chopped into shorter lengths using sonication dispersion and these 
tubes are individually dispersed without severe aggregation.

3.2 Electron transfer properties of PANI nanotube ensembles as assessed by cyclic voltammetry

Ensembles of PANI nanomaterial are commonly used in electrochemical and electrical devices.[4, 10] We therefore have studied the redox reaction of the PANI nanotube ensembles formed by the drop-casting of a PANI suspension onto the surface of GCE. Upon immersing the modified electrode in $0.2 \mathrm{M} \mathrm{H}_{2} \mathrm{SO}_{4}$, a stable open circle potential (OCP) was recorded before applying any potential. Subsequently, the CV scan was commenced from the OCP of the nanotube coated GCE and the first scan recorded, thus ensuring the electrochemical reaction starts from the original metallic emeraldine salt form of PANI nanotubes. Fig. 3A exhibits the typical CV curves of the PANI nanotube modified GCE with various amounts of coating. As expected, two pairs (1, 1' and 2, 2') of redox peaks are found, which are assigned to the electrolytic doping/dedoping process associated with the electron transfer process. A 2-electron transfer occurs in each redox reaction for one repeat unit (composed of 4 anilines). The reduction and oxidation of the emeraldine salt on the modified electrode surface leads to the formation of Leuco and the Per forms, respectively, as described schematically in Fig. 1.

In order to estimate the density and thickness of the PANI nanotubes coated on the electrode surface, the mass of material drop-cast on the electrode was converted into an average number of monolayers. Assuming the PANI tubes lie in parallel on the electrode surface, the number $\left(N_{l}\right)$ of layers of the tubes is calculated based on the following equation:

$\mathrm{N}_{l l}$ 
und $W_{\text {tumber }}-$ 
where, $c\left(g L^{-1}\right)$ is the concentration of PANI suspension, $V_{\text {sol }}$ is the drop volume on electrode, $A$ is the geometric surface area of the electrode, $L$ and $d$ is the length and outmost diameter of the tube, respectively. $V_{\text {tube }}$ and $\rho\left(1.33 \times 10^{6} \mathrm{~g} \mathrm{~m}^{-3}\right)$ [42] represent the volume of a single tube and the material density, respectively.

The magnitude of the PANI voltammetric response is found to scale with the mass coated in Fig. 3A. Peak 1 represents the reduction of original emeraldine PANI, and this peak is used to quantify the charge transferred to the PANI nanotube. The average charges for the different number of layers are shown in Fig. 3B. A linear relationship between the charge and the layer number is obtained. Moreover, from this the average charge passed per an individual tube can be calculated. As shown in the inlay of Fig. 3B, at various deposition amounts the charge transferred is almost identical, with a mean charge of $(10.01 \pm 0.94) \mathrm{pC}$ per tube. The theoretical charge value of one tube may be estimated according to the following equation (2),

$$
Q Q_{\text {ttububee }}=\frac{\text { pol } V_{\text {tumbee }}}{\text { umminitt }}
$$

where $Q_{\text {tube }}$ is the total charge on an individual tube, $V_{\text {tube }}$ and $\rho$ are as defined earlier. $M_{\text {unit }}$ and $n$ are the molecular weight (435 $\left.\mathrm{g} \mathrm{mol}^{-1}\right)$ and electron transfer number of each repeat unit (composed of 4 anilines) in a redox reaction, respectively. $n$ equals 2 according to Fig. $1 . \mathrm{N}$ and e are the Avogadro constant and the elemental charge (= $\left.1.602 \times 10^{-19} \mathrm{C}\right)$, respectively. Based on the mean tube size found from SEM, the expected charge of an individual tube is calculated to be $(171 \pm 112)$ pC. Consequently in comparison to the value experimentally measured using cyclic voltammetry it is concluded that about $6 \%$ of PANI tube participates in the redox reaction on a macroelectrode. The significantly lower doping level may physically arise due to two reasons. One is the inevitable aggregation of the PANI tubes on the electrode surface[33, 34]. The other is that there is conductivity loss upon oxidation or 
reduction of PANI due to the transition of the metallic conductive emeraldine salt into the insulating oxidation/reduction state. Therefore, only materials in direct contact with the electrode surface contribute to the charge transfer. Since the doping level of each tube is ambiguous using the drop-cast technique, the nano-impact method which enables studying single nanotube is applied in the following section.

\subsection{Electron transfer to and from single PANI nanotubes}

With the aim of characterizing the extent of electron transfer to/from individual PANI nanotubes, nano-impact experiments were performed using a carbon micro wire working electrode via chronoamperometry. Chronoamperograms were recorded in a deaerated $0.2 \mathrm{M}$ $\mathrm{H}_{2} \mathrm{SO}_{4}$ aqueous solution containing $0.01 \mathrm{~g} \mathrm{~L}^{-1}$ PANI nanotube at various applied potentials with the duration time of 50s. Representative chronoamperograms at $-0.2 \mathrm{~V}$ and $+0.8 \mathrm{~V}$ are shown in Fig. 4A and 4B, respectively. Typical reduction and oxidation spikes are magnified and shown in the inset with the corresponding curves, which is consistent with the reduction and oxidation of the conductive 'half-oxidized' emeraldine PANI observed in the cyclic voltammograms. For comparison, the chronoamperograms were recorded at $-0.2 \mathrm{~V}$ and +0.8 $\mathrm{V}$ in $0.2 \mathrm{M} \mathrm{H}_{2} \mathrm{SO}_{4}$ in the absence of PANI nanotubes. No spikes in the current transients were observed (red lines). A total of 183 spikes were observed at $-0.2 \mathrm{~V}$ over 101 scans (50s span in each scan) in $0.01 \mathrm{~g} \mathrm{~L}^{-1}$ PANI. The collision frequency is near $0.036 \mathrm{~Hz}$, which is in good agreement with the value estimated from the use of the Stokes-Einstein-Sutherland equation and the diffusion-limited transport model $(0.038 \mathrm{~Hz})[43,44]$. This corroborates the conclusion that the current spikes are from the electron transfer of a single PANI nanotube upon collision with the micro electrode.

The charge passed during the collision between nanotube and micro electrode was determined by integrating the area under each individual transient signal. The histograms of 
the integrated charges under transient signals collected at $-0.2 \mathrm{~V}$ and $+0.8 \mathrm{~V}$ show a lognormal distribution (Fig. S2A and S2B), with the mean charge value of $31.5 \pm 6.7 \mathrm{pC}$ and $14.3 \pm 6.7 \mathrm{pC}$ at $-0.2 \mathrm{~V}$ and $+0.8 \mathrm{~V}$, respectively.

To find the influence of the electrode potential on the impact experiment, chronoamperograms were recorded at different potentials from $-0.4 \mathrm{~V}$ to $+1.0 \mathrm{~V}$, in $0.2 \mathrm{M}$ $\mathrm{H}_{2} \mathrm{SO}_{4}$ aqueous solution containing $0.01 \mathrm{~g} \mathrm{~L}^{-1} \mathrm{PANI}$ nanotube. The charge passed at all applied potentials was found to show a log-normal distribution. The mean charges at different potentials are summarized in Fig. 5 (the numbers of spikes at each potential are labelled inside Fig. 5). No spikes were observed in the potential range of $0.1 \sim 0.3 \mathrm{~V}$. Therefore, a charge value of $0 \mathrm{pC}$ is given at this potential range. In the reduction zone, the average charge increases obviously with the negative shift of the applied potential from $0 \mathrm{~V}$ to $-0.2 \mathrm{~V}$, owing to the increased driving force for the electrochemical reduction of emeraldine salt. The average charge shows a maximum at $-0.2 \mathrm{~V}$. With shifting the potential more negatively, the corresponding charge is found to decrease (this is explained below). A similar trend is found in oxidation, but a lower charge is obtained.

For comparison, a typical CV curve of PANI nanotube ensembles on GCE was overlaid as shown in Fig. 5 (blue curve). The threshold potential ( $0 \mathrm{~V}$, at the reduction potential zone) of a single nanotube impact in chronoamperometry shifts negatively, in comparison with the onset potential ( $\sim 0.3 \mathrm{~V}$ ) of PANI nanotube ensembles. The variation can be attributed to the very short duration time (20-30 ms) of individual impact spike which requires higher overpotential to instantly drive the redox reaction of PANI upon collision. Similar phenomena were observed in other nano-impact systems [45].

The obtained charge maximum input $(31.5 \pm 6.7 \mathrm{pC})$ at a single nanotube as measured via the nano-impact method is much larger than that for an ensemble of tubes on a 
macroelectrode $(10.01 \pm 0.84 \mathrm{pC})$. However, still only ca. $18 \%$ of the PANI tubes participate in the charge transfer reaction compared with the estimated maximum value of $(171 \pm 112)$ pC. This result further confirms that the loss of electrical contact due to the reduction/oxidation of metallic $\mathrm{Em} \cdot \mathrm{HCl}$ results in the incomplete charge transfer. It is surmised that at lower overpotentials, the charge transfer of the PANI is slow and electron can be transferred further along the tube before it is transformed into the insulating state. In contrast to at higher overpotentials $(\leq-0.3 \mathrm{~V})$, charge transfer is very fast. Upon collision to the wire electrode, only the part of PANI nanotube in direct contact with the wire electrode is totally transformed into the non-conducting state. This results in the loss of the electrical contact of the tube with the electrode, so that the electron transfer is blocked for the remaining part of the PANI tube.

\section{Conclusions}

The nano-impact technique has been used for the first time to study the electrochemical doping extent of a single PANI nanotube in aqueous sulphuric acid solution. The charge transfer is more than doubled by using single tube collisions with carbon fibre micro wire electrode, compared with the doping of tube ensembles. However still only partial doping $(<18 \%)$ of PANI was observed due to the loss of the electrical contact during the transition of metallic emeraldine salt to insulating PANI. These observations have significant implications for the use of PANI nanomaterials applications requiring electrical conductivity.

\section{Acknowledgments}

This research was supported by the European Research Council (ERC) under the European Union's Seventh Framework Programme (FP/2007-2013), ERC Grant Agreement no.320403. National Natural Science Foundation of China (No. 21476047) is gratefully acknowledged. J. Liu and X. Li thank for the support of the China Scholarship Council. 


\section{References:}

[1] M. Xin, H. Lin, J. Yang, M. Chen, X. Ma, J. Liu, Preparation of polyaniline/auo nanocomposites modified electrode and application for hydrazine detection, Electroanalysis, 26 (2014) 2216-2223.

[2] Yu Gao, Decai Shan, Fei Cao, Jian Gong, Xia Li, Hui-yan Ma, Zhong-min Su, L.-y. Qu, Silver/polyaniline composite nanotubes: one-step synthesis and electrocatalytic activity for neurotransmitter dopamine, J. Phys. Chem. C, 113 (2009) 15175-15181.

[3] X. Ma, J. Yang, W. Cai, G. Zhu, J. Liu, Preparation of au nanoparticles decorated polyaniline nanotube and its catalytic oxidation to ascorbic acid, Chemical Research in Chinese Universities, 32 (2016) 702-708.

[4] S. Bao, M. Du, M. Zhang, H. Zhu, P. Wang, T. Yang, M. Zou, Facile fabrication of polyaniline nanotubes/gold hybrid nanostructures as substrate materials for biosensors, Chemical Engineering Journal, 258 (2014) 281-289.

[5] S.K. Simotwo, V. Kalra, Polyaniline-based electrodes: recent application in supercapacitors and next generation rechargeable batteries, Current Opinion in Chemical Engineering, 13 (2016) 150-160.

[6] A. Mirmohseni, R. Solhjo, Preparation and characterization of aqueous polyaniline battery using a modified polyaniline electrode, European Polymer Journal, 39 (2003) 219-223. [7] Q.L. Hao, X.F. Xia, W. Lei, W.J. Wang, J.S. Qiu, Facile Synthesis of Sandwich-Like Polyaniline/Boron-Doped Graphene Nano Hybrid for Supercapacitors, Carbon, 81 (2015) $552-563$

[8] Y.-E. Miao, W. Fan, D. Chen, T. Liu, High-Performance Supercapacitors based on hollow polyaniline nanofibers by electrospinning, ACS Applied Materials \& Interfaces, 5 (2013) 4423-4428.

[9] Z.-L. Wang, R. Guo, G.-R. Li, H.-L. Lu, Z.-Q. Liu, F.-M. Xiao, M. Zhang, Y.-X. Tong, Polyaniline nanotube arrays as high-performance flexible electrodes for electrochemical energy storage devices, Journal of Materials Chemistry, 22 (2012) 2401.

[10] D. Vonlanthen, P. Lazarev, K.A. See, F. Wudl, A.J. Heeger, A stable polyanilinebenzoquinone-hydroquinone supercapacitor, Advanced Materials, 26 (2014) 5095-5100.

[11] F.A. Rafiqi, K. Majid, Synthesis, Characterization, photophysical, thermal and electrical properties of composite of polyaniline with zinc bis (8-hydroxyquinolate): a potent composite for electronic and optoelectronic use, RSC Advances, 6 (2016) 22016-22025. 
[12] C.-H. Chang, T.-C. Huang, C.-W. Peng, T.-C. Yeh, H.-I. Lu, W.-I. Hung, C.-J. Weng, T.-I. Yang, J.-M. Yeh, Novel anticorrosion coatings prepared from polyaniline/graphene composites, Carbon, 50 (2012) 5044-5051.

[13] N. Sarkar, G. Sahoo, R. Das, G. Prusty, D. Sahu, S.K. Swain, Anticorrosion performance of three-dimensional hierarchical PANI@BN nanohybrids, Industrial \& Engineering Chemistry Research, 55 (2016) 2921-2931.

[14] P.C. Rodrigues, L. Akcelrud, Networks and blends of polyaniline and polyurethane: correlations between composition and thermal, dynamic mechanical and electrical properties, Polymer, 44 (2003) 6891-6899.

[15] A. Pande, P. Gairola, P. Sambyal, S. Gairola, V. Kumar, K. Singh, S. Dhawan, Electromagnetic shielding behavior of polyaniline using Red Mud (industrial waste) as filler in the X-band (8.2-12.4 GHz) frequency range, Materials Chemistry and Physics, 189 (2017) 22-27.

[16] W.-S. Huang, B.D. Humphrey, A.G. MacDiarmid, Polyaniline, a novel conducting polymer. Morphology and chemistry of its oxidation and reduction in aqueous electrolytes, $\mathrm{J}$. Chem. Soc., Faraday Trans., 82 (1986) 2385-2400.

[17] A.G. MacDiarmid, A.J. Epstein, Polyanilines: a novel class of conducting polymers, Faraday Discussions of the Chemical Society, 88 (1989) 317-332.

[18] A.G. MacDiarmid, "Synthetic metals": a novel role for organic polymers (Nobel lecture), Angewandte Chemie International Edition, 40 (2001) 2581-2590.

[19] J.-C. Chiang, A.G. MacDiarmid, 'Polyaniline’: Protonic acid doping of the emeraldine form to the metallic regime, Synthetic Metals, 13 (1986) 193-205.

[20] A. MacDiarmid, J. Chiang, A. Richter, Epstein, AJ, Polyaniline: a new concept in conducting polymers, Synthetic Metals, 18 (1987) 285-290.

[21] T. Hjertberg, W. Salaneck, I. Lundstrom, N. Somasiri, A. MacDiarmid, A 13C CP - MAS NMR investigation of polyaniline, Journal of Polymer Science: Polymer Letters Edition, 23 (1985) 503-508.

[22] J. Jacq, Schema carre: Etablissement et discussion de l'equation generale de la courbe intensite-potentiel en regime stationnaire et diffusion convective, Journal of Electroanalytical Chemistry and Interfacial Electrochemistry, 29 (1971) 149-180.

[23] C. Batchelor-McAuley, Q. Li, S.M. Dapin, R.G. Compton, Voltammetric characterization of DNA intercalators across the full $\mathrm{pH}$ range: Anthraquinone-2, 6- 
disulfonate and anthraquinone-2-sulfonate, The Journal of Physical Chemistry B, 114 (2010) 4094-4100.

[24] B.J. Gallon, R.W. Kojima, R.B. Kaner, P.L. Diaconescu, Palladium nanoparticles supported on polyaniline nanofibers as a semi-heterogeneous catalyst in water, Angewandte Chemie, 46 (2007) 7251-7254.

[25] C.O. Baker, B. Shedd, R.J. Tseng, A.A. Martinez-Morales, C.S. Ozkan, M. Ozkan, Y. Yang, R.B. Kaner, Size control of gold nanoparticles grown on polyaniline nanofibers for bistable memory devices, ACS nano, 5 (2011) 3469-3474.

[26] D. Li, J. Huang, R.B. Kaner, Polyaniline nanofibers: a unique polymer nanostructure for versatile applications, Accounts of chemical research, 42 (2008) 135-145.

[27] P. Anilkumar, M. Jayakannan, Hydroxyl-functionalized polyaniline nanospheres: Tracing molecular interactions at the nanosurface via vitamin C sensing, Langmuir : the ACS journal of surfaces and colloids, 24 (2008) 9754-9762.

[28] Y. Yang, Y. Chu, F. Yang, Y. Zhang, Uniform hollow conductive polymer microspheres synthesized with the sulfonated polystyrene template, Materials Chemistry and Physics, 92 (2005) 164-171.

[29] H. Dong, S. Prasad, V. Nyame, W.E. Jones, Sub-micrometer conducting polyaniline tubes prepared from polymer fiber templates, Chemistry of materials, 16 (2004) 371-373. [30] E.N. Konyushenko, J. Stejskal, I. Šeděnková, M. Trchová, I. Sapurina, M. Cieslar, J. Prokeš, Polyaniline nanotubes: conditions of formation, Polymer International, 55 (2006) 3139.

[31] H. Qiu, M. Wan, B. Matthews, L. Dai, Conducting polyaniline nanotubes by templatefree polymerization, Macromolecules, 34 (2001) 675-677.

[32] G.G. Wildgoose, C.E. Banks, H.C. Leventis, R.G. Compton, Chemically modified carbon nanotubes for use in electroanalysis, Microchimica Acta, 152 (2006) 187-214.

[33] H.S. Toh, C. Batchelor-McAuley, K. Tschulik, M. Uhlemann, A. Crossley, R.G. Compton, The anodic stripping voltammetry of nanoparticles: electrochemical evidence for the surface agglomeration of silver nanoparticles, Nanoscale, 5 (2013) 4884-4893.

[34] S.J. Cloake, H.S. Toh, P.T. Lee, C. Salter, C. Johnston, R.G. Compton, Anodic stripping voltammetry of silver nanoparticles: aggregation leads to incomplete stripping, ChemistryOpen, 4 (2015) 22-26. 
[35] H.S. Toh, K. Jurkschat, R.G. Compton, The Influence of the capping agent on the oxidation of silver nanoparticles: nano - impacts versus stripping voltammetry, Chemistry A European Journal, 21 (2015) 2998-3004.

[36] W. Cheng, R.G. Compton, Electrochemical detection of nanoparticles by 'nano-impact' methods, TrAC Trends in Analytical Chemistry, 58 (2014) 79-89.

[37] S.V. Sokolov, S. Eloul, E. Katelhon, C. Batchelor-McAuley, R.G. Compton, Electrodeparticle impacts: a users guide, Physical chemistry chemical physics : PCCP, 19 (2016) 28-43. [38] P.H. Robbs, N.V. Rees, Nanoparticle electrochemistry, Physical chemistry chemical physics : PCCP, 18 (2016) 24812-24819.

[39] N.V. Rees, Electrochemical insight from nanoparticle collisions with electrodes: A minireview, Electrochemistry Communications, 43 (2014) 83-86.

[40] H. Wu, Q. Lin, C. Batchelor - McAuley, R.G. Compton, Nanoimpacts reveal the electron - transfer kinetics of the ferrocene/ferrocenium couple immobilised on graphene nanoplatelets, ChemElectroChem, 3 (2016) 1478-1483.

[41] J. Ellison, C. Batchelor-McAuley, K. Tschulik, R.G. Compton, The use of cylindrical micro-wire electrodes for nano-impact experiments; facilitating the sub-picomolar detection of single nanoparticles, Sensors and Actuators B: Chemical, 200 (2014) 47-52.

[42] J. Stejskal, R. Gilbert, Polyaniline. Preparation of a conducting polymer (IUPAC technical report), Pure and Applied Chemistry, 74 (2002) 857-867.

[43] S. Eloul, E. Kätelhön, C. Batchelor-McAuley, K. Tschulik, R.G. Compton, Diffusional impacts of nanoparticles on microdisc and microwire electrodes: the limit of detection and first passage statistics, Journal of Electroanalytical Chemistry, 755 (2015) 136-142.

[44] W. Sutherland, LXXV. A Dynamical theory of diffusion for non-electrolytes and the molecular mass of albumin. The London, Edinburgh, and Dublin Philosophical Magazine and Journal of Science, 9(54) (1905): 781-785.

[45] H. Wu, Q. Lin, C. Batchelor-McAuley, L.M. Goncalves, C.F. Lima, R.G. Compton, Stochastic detection and characterisation of individual ferrocene derivative tagged graphene nanoplatelets, The Analyst, 141 (2016) 2696-2703. 


\section{Figure captions:}

Fig. 1. (A) Diagram showing the different protonation and oxidation degrees of PANI. The horizontal direction in the square represents the degree of oxidation, and the vertical direction represents the degree of protonation; (B) PANI formulae with y referring to the different oxidation states ; (C) Radical cation chain structure of Em· HCl. The number of repeat unit $n$ is equal to 4 monomers.

Fig. 2. (A) SEM image and cross-section (upper left inlay) image of PANI hollow fibres; Inlay in lower left of (A) is a TEM image of a PANI nanotube. (B) SEM image of chopped PANI nanotubes drop-cast on ITO substrate. (C) and (D) are the length and width distributions of PANI nanotubes, respectively. The mean lengths and widths are found to be $2.89 \pm 1.9 \mu \mathrm{m}$ and $0.39 \pm 0.06 \mu \mathrm{m}$, respectively.

Fig. 3. (A) Cyclic voltammograms of PANI ensembles on GCE with various dropping amount (from inside to outside: $1,2,5,8$ and $15 \mu \mathrm{L}$ ) in $0.2 \mathrm{M} \mathrm{H}_{2} \mathrm{SO}_{4}$ solution at a scan rate of $100 \mathrm{mV} / \mathrm{s}$; (B) Total charge on different layers (corresponding to 1, 2, 5, 8 and $15 \mu \mathrm{L}$ drop amount, respectively). Inset of (B) is the charge per tube for different number of layers.

Fig. 4. Representative chronoamperometric scans in $0.01 \mathrm{~g} \mathrm{~L}^{-1} \mathrm{PANI}$ nanotube suspension at a cylindrical carbon fibre wire electrode applying a potential of $-0.2 \mathrm{~V}(\mathrm{~A})$ and $0.8 \mathrm{~V}(\mathrm{~B})$. Red lines: blank scan in $0.2 \mathrm{M} \mathrm{H}_{2} \mathrm{SO}_{4}$ solution. Inset is the characteristic spike at each corresponding potential.

Fig. 5. The average charge of the spikes as a function of applied potential. Labelled are the numbers of spikes. The blue line is the CV curve of PANI ensembles on GCE. 
Fig. 1.
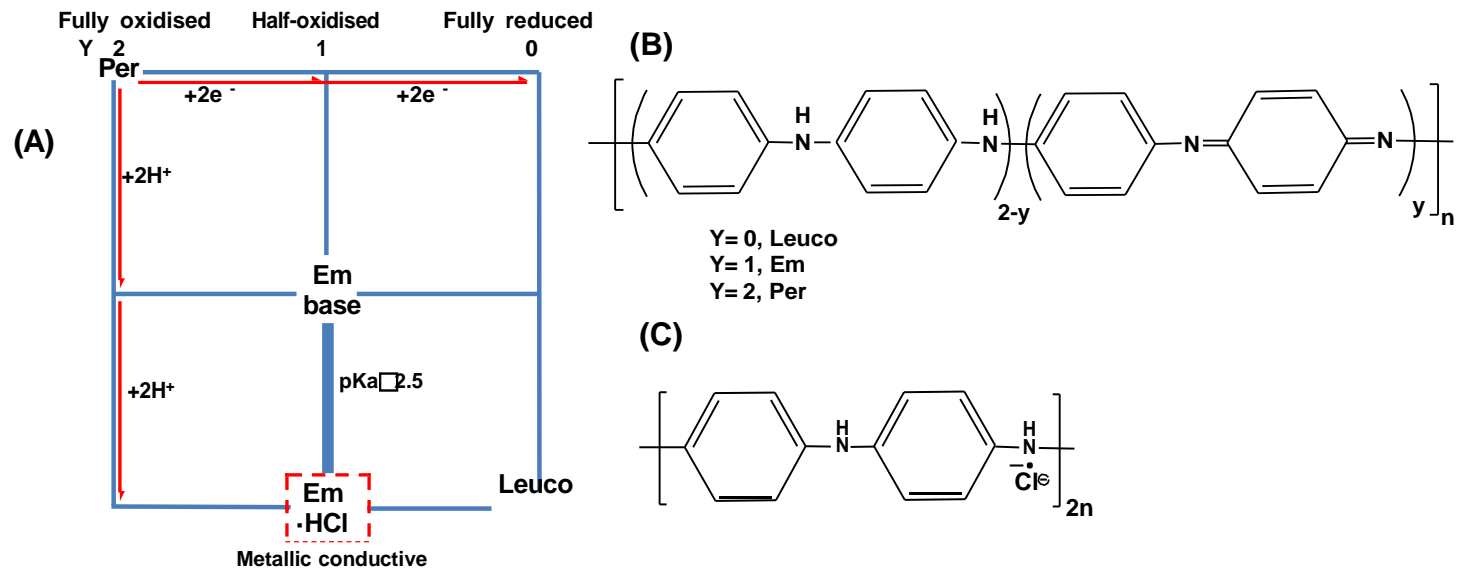

(C)

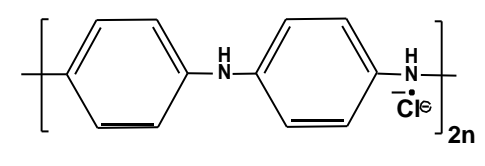


Fig. 2.
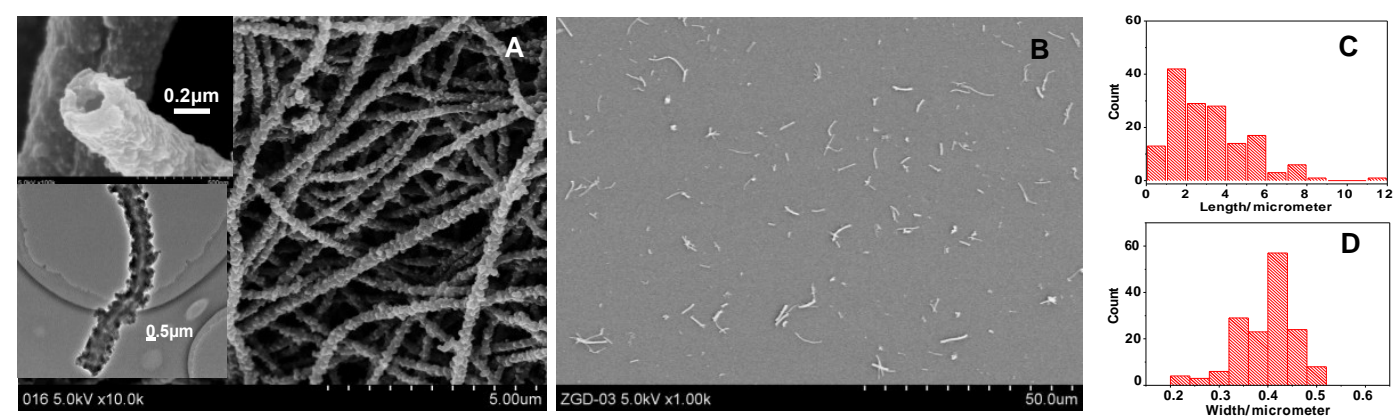
Fig. 3.
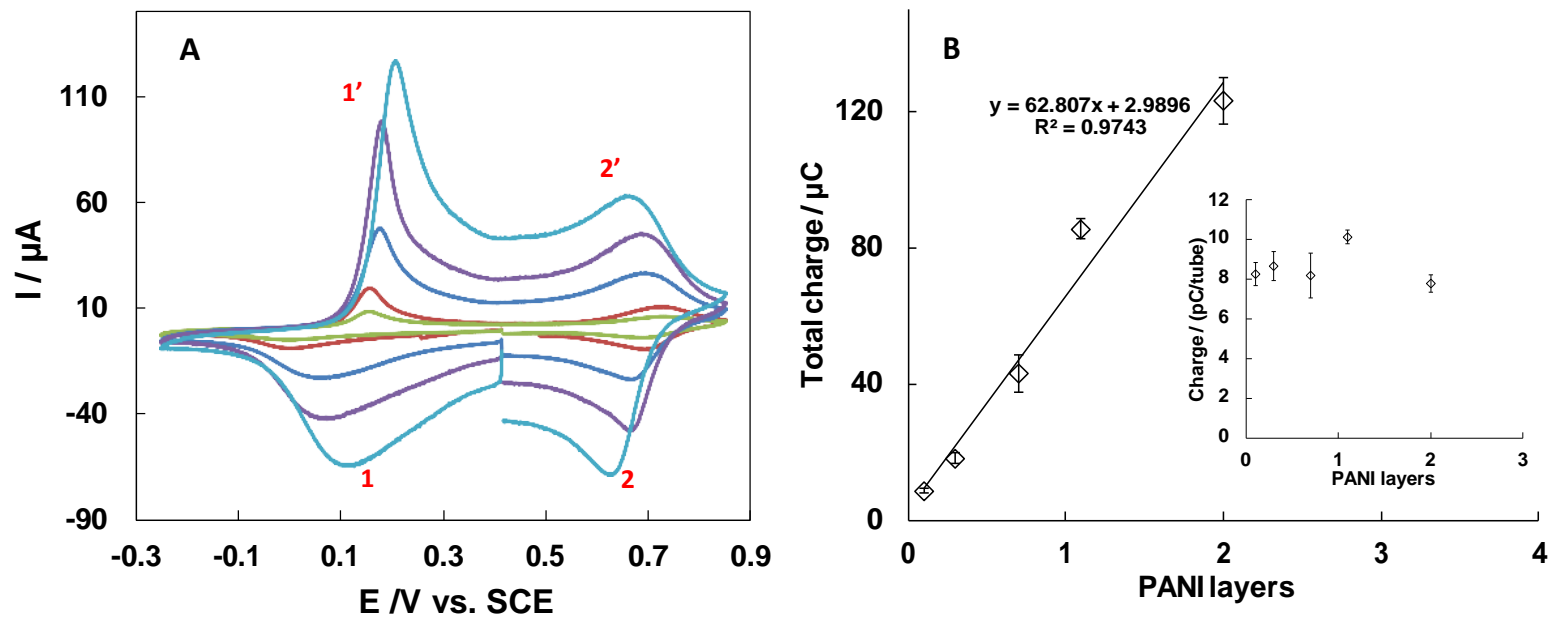
Fig. 4.
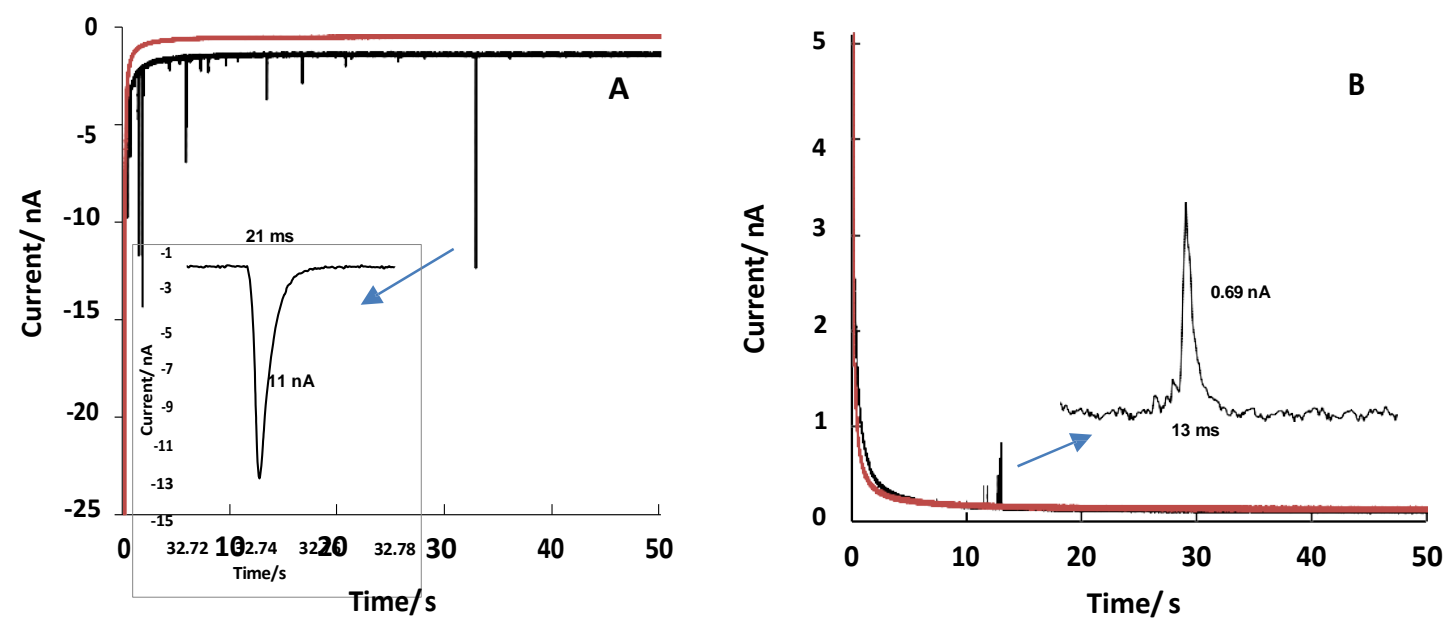
Fig. 5.

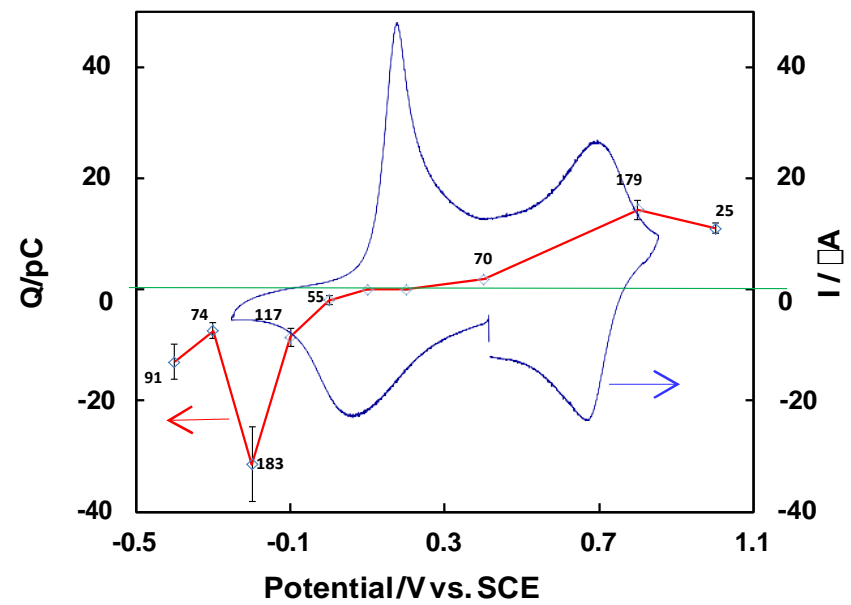




\section{Estimation of inner diameter of PANI nanotube}

The SEM image of the electrospun polystyrene fibres is shown in Fig. S1. The average diameter was calculated to be $156 \pm 30 \mathrm{~nm}$ based on 30 fibers.
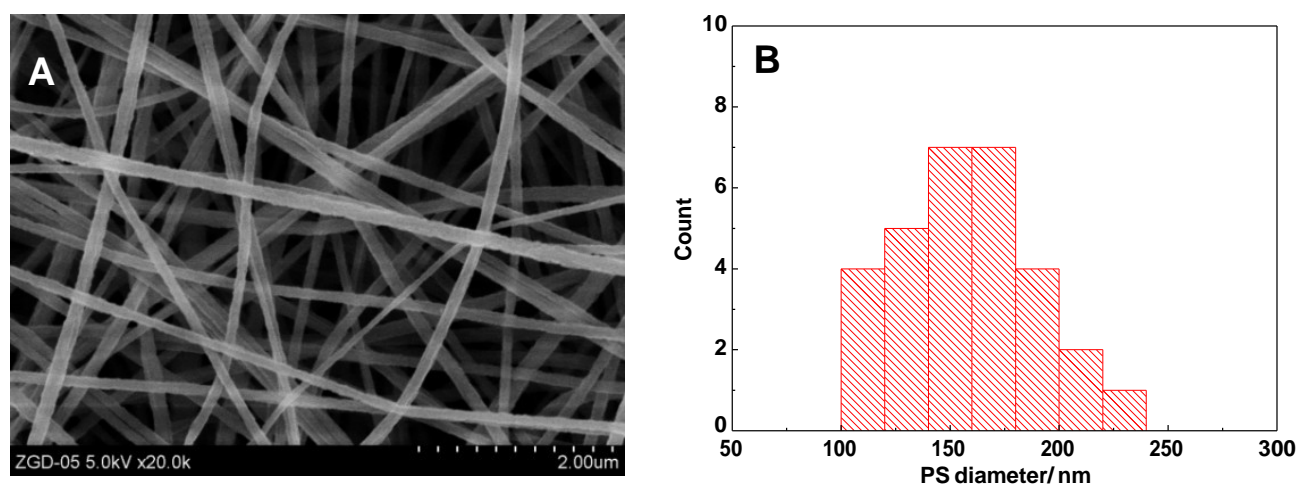

Figure S1 (A) SEM image of the electrospun polystyrene fibres; (B) Distribution of diameter of the fibres

\section{Charge distribution derived from integration of spikes at different voltage}
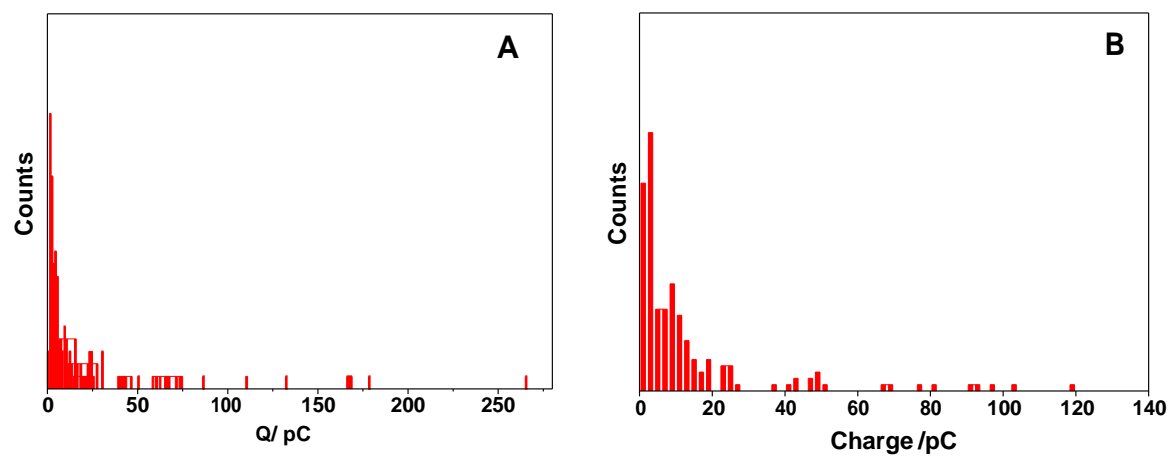

Figure S2 Distribution of the charge extracted from spikes resulted from chronoamperometric measurements in $\mathrm{N}_{2}$-saturated $\mathrm{H}_{2} \mathrm{SO}_{4}$ solution $(0.2 \mathrm{M})$ at $-0.2 \mathrm{~V}(\mathrm{~A})$ and $0.8 \mathrm{~V}(\mathrm{~B})$, respectively. 


\section{Highlights:}

- The doping level of a single PANI nanotube is quantified by nano-impact method

- Electrochemical doping of a single PANI nanotube and tube ensembles is compared

- Electron transfer on a single tube is 3 times higher than that on the ensembles

- Only partial doping (18\%) of the tubes is achieved even by nano-impact technique 